\title{
Spiral Evolution in a Confined Geometry
}

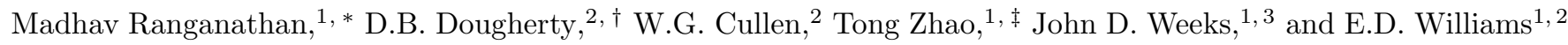 \\ ${ }^{1}$ Institute of Physical Sciences and Technology, University of Maryland, College Park, MD 20742-2431. \\ ${ }^{2}$ Materials Research Science and Engineering Center, Department of Physics, \\ University of Maryland, College Park, MD 20742-4111. \\ ${ }^{3}$ Department of Chemistry and Biochemistry, University of Maryland, College Park, MD 20742-4454.
} (Dated: December 11, 2018)

\begin{abstract}
Supported nanoscale lead crystallites with a step emerging from a non-centered screw dislocation on the circular top facet were prepared by rapid cooling from just above the melting temperature. STM observations of the top facet show a nonuniform rotation rate and shape of the spiral step as the crystallite relaxes. These features can be accurately modeled using curvature driven dynamics, as in classical models of spiral growth, with boundary conditions fixing the dislocation core and regions of the step lying along the outer facet edge.
\end{abstract}

PACS numbers: 05.20.-y, 61.72.Ff, 68.35.-p, 68.37.Ef

The control of mass flow in the fabrication and evolution of nanoscale structures is a key problem in nanoscience. Scanned probe techniques can be used to directly observe dynamical evolution, permitting the development of detailed models that can yield a predictive understanding of nanostructure evolution $1,2,2,3,4,5,6$. 6 . Here we address a classical problem - the facilitation of structural evolution by the presence of screw dislocations - in a novel context where the spiral step is confined to the top facet of a supported crystallite island prepared in a non-equilibrium configuration.

Frank predicted that screw dislocations serve as a continuous source of steps [7, 8] crucial for step-flow growth on nominally flat terraces. In the presence of a supersaturation in the vapor, an infinitely long straight step starting at a dislocation rapidly winds into a spiral step. Once the curvature of the step at the dislocation core attains a particular critical value corresponding to the size of the critical nucleus in classical nucleation theory, the entire spiral rotates uniformly, with an angular velocity determined by the supersaturation of the vapor and the material parameters. Such growth has been studied by experiments 9, 10], theory 8, 11, 12, 13] and Monte Carlo simulations 14]. These studies of spiral evolution have dealt with processes on infinite substrates. We show here how the dynamics of spiral steps in confined geometries can be appropriately modeled by accounting for the boundary conditions imposed by the edges of the nanocrystalline structures.

We prepared three dimensional crystallites of $\mathrm{Pb}$ on a $\mathrm{Ru}(0001)$ substrate using methods described in previous work [6, 15]. Direct observation of the relaxation of the crystallites following a rapid change in temperature was achieved using variable temperature scanning tunneling microscopy (VT-STM) [3, 5]. By controlling the rate of cooling from $550 \mathrm{~K}$ to the measurement temperature of $390 \mathrm{~K}$, it is possible to create crystal populations where about $30 \%$ of the structures have a screw dislocation on the top facet that is embedded many layers into the crystal. Crystallites with such dislocations have been shown to relax to a final state much closer to the expected equilibrium crystal shapes (ECS) than those without dislocations, suggesting that dislocations provide an easier kinetic pathway to crystal relaxation 16, 17, 19.

As shown in Fig. 1h, the shape of the step emerging from the dislocation qualitatively resembles the initial shape of a normal growth spiral. However, the later turns of the spiral merge into the edge of the crystallite instead of forming the expanding shape of a growth spiral. A geometrical model describing the structure of the spiral is shown in Fig 1b. Time resolved STM imaging of the structure as illustrated by Fig 2 a shows that the spiral turns rapidly in the uphill direction, opposite to that of a growth spiral, corresponding to mass leaving the step edge. The time resolved measurements show strikingly that the shapes and angular velocities change substantially during each turn of the spiral step as successive layers of the crystallite peel off. In particular the motion of the step becomes so fast that only a few frames are captured 20] as the angle $\theta_{0}$, as defined in Fig 10, changes from $\pi$ to $2 \pi$. The radius of the top facet increases only very slowly compared to the motion of the spiral step and remains essentially constant for many turns of the spiral. To model this behavior, we assume, as in previous models of spiral growth 8, 21], that the step starts at a fixed point that corresponds physically to the core of the dislocation and moves according to the usual laws of motion for isolated steps. Here however the extent of the step is limited by the finite size of the island. We model this as a boundary condition confining the step to a fixed outer circle representing the island boundary. We assume there is no transport of atoms through the bulk and neglect the anisotropy of the step stiffness. The experimental conditions and the low vapor pressure of $\mathrm{Pb}$ justify these assumptions.

The local chemical potential $\mu$ of the curved step relative to a straight step can be written down using the Gibbs-Thompson relation 22] $\mu=\Omega_{s} \tilde{\beta} \kappa$, where $\kappa$ is the 

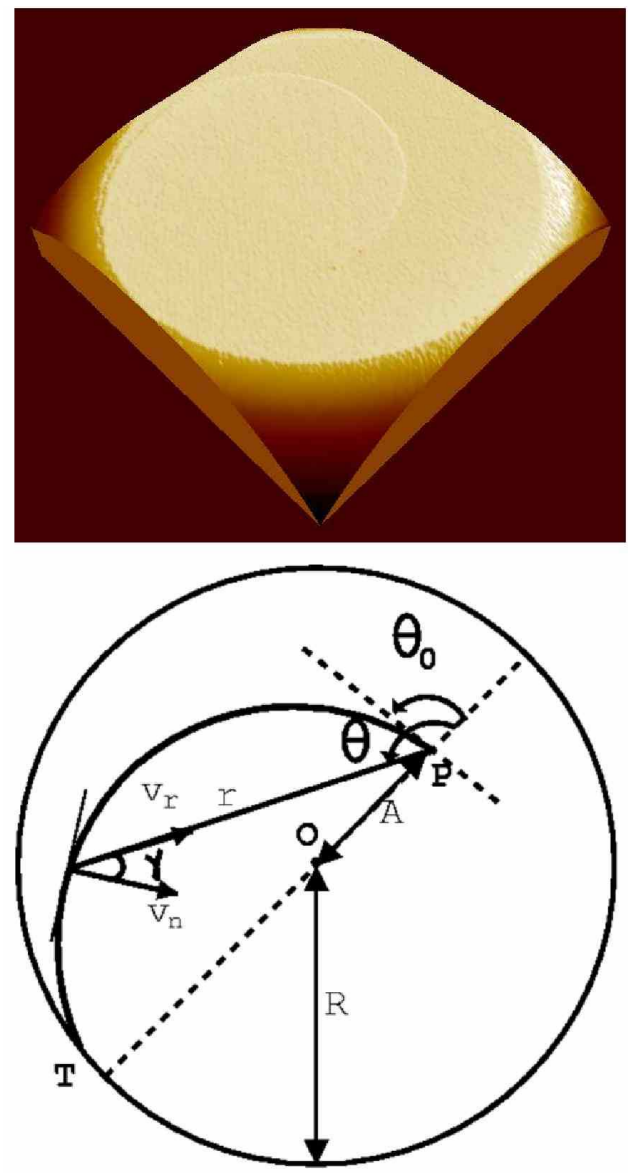

FIG. 1: color on-line(a) STM picture of a 3-D crystallite of $\mathrm{Pb}$ on $\mathrm{Ru}$ with a screw dislocation leading to a spiral step on the top facet. (b) The top facet of a crystallite containing an embedded screw dislocation is modeled as a circle of radius $R$. The dislocation step is shown as the bold curve that begins at point $\mathrm{P}$, which is a distance $A$ from the center of the circle 0 , and joins the circle smoothly at the joining point $\mathrm{T}$. Beyond T, the dislocation step follows along the circle in the counterclockwise direction. The shape of the step for a given initial angle $\theta_{0}$ is specified by the distance $r$ as a function of the angle $\theta$ along the curve. The directions of the radial velocity of the step $v_{r}$ and its component normal to the step $v_{n}$ are separated by an angle $\gamma$.

local curvature of the step, $\Omega_{s}$ is the atomic area and $\tilde{\beta}$ the step stiffness. The step velocity normal to the local direction of the step is denoted by $v_{n}$ and can be derived from the chemical potential for different limiting cases. We use a model where the rate limiting process is attachment and detachment of atoms from the step region 23. Then, the local step segment moves according to the chemical potential difference between the step and a reservoir chemical potential maintained by fast diffusion on the terrace. The local step velocity is given by $v_{n}(\kappa)=\left(\Gamma_{A} / \Omega_{s} k T\right)\left[\mu(\kappa)-\mu_{\text {res }}\right]$ where $\Gamma_{A}$ is the mobility of the step edge and $\mu_{\text {res }}$ is the chemical potential of the reservoir. Substituting for $\Gamma_{A}$ in terms of the rate

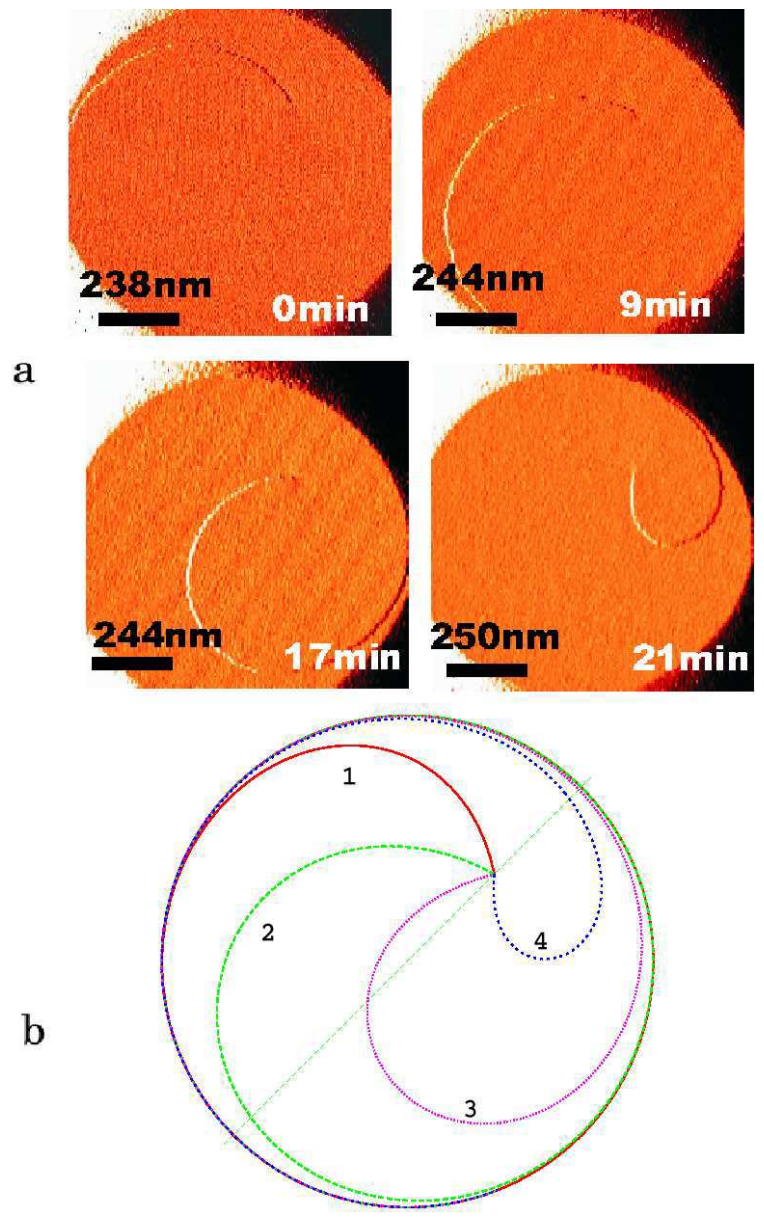

FIG. 2: color on-line(a)STM snapshots of the time evolution of the step due to the dislocation, (b) corresponding snapshots at time $t$ of the calculated evolution superimposed onto a single figure. Curve 1 (red) $t=0$, curve 2 (green) $t=(9 / 23) T$, curve 3 (violet) $t=(17 / 23) T$, curve 4 (blue) $t=(21 / 23) T$, where $T$ is the period of rotation.

constant for detachment from the step edge $k_{A D}$ 22, 24], the equilibrium concentration of adatoms on the terrace $c_{e q}$ and the lattice constant $a$ yields the following expression for the local normal step velocity in terms of the local curvature:

$$
v_{n}(\kappa)=\frac{a^{4} c_{e q} \tilde{\beta} k_{A D}}{k T}\left[\kappa-\kappa_{c}\right]
$$

In the above equation, we have expressed the reservoir chemical potential in terms of a cutoff curvature $\kappa_{c}$. The atoms detaching from the dislocation step diffuse across the top facet to the edge of the crystallite, and down its sides, causing motion of the crystallite edge 25]. However, the time scale of this motion is much larger than that of the motion of the dislocation step during each turn. Thus, to describe motion of the dislocation step on the top facet, the edge of the crystallite can be treated as an effective reservoir with $\kappa_{c}=1 / R$ where $R$ is the average radius of the top facet. We assume that the spi- 
ral step smoothly joins this outer circle, which with our choice of $\kappa_{c}$ does not move as the evolution proceeds.

Figure 1 describes the coordinate system that we will use for our analysis. In these coordinates, the curvature is given by

$$
\kappa=\frac{r^{2}+2{r^{\prime}}^{2}-r r^{\prime \prime}}{\left(r^{2}+{r^{\prime}}^{2}\right)^{3 / 2}}
$$

where the prime denotes a derivative with respect to the angle $\theta$. The limiting angle $\theta_{0}$ as the step emerges from the core has $r\left(\theta_{0}\right)=0$ and Eq. (2) then gives $r^{\prime}\left(\theta_{0}\right)=$ $2 / \kappa\left(\theta_{0}\right)$. We can take $r(\theta)=r^{\prime}(\theta)=0$ for $0 \leq \theta \leq \theta_{0}$. Thus $\theta_{0}$ is the smallest angle at which $r^{\prime}(\theta) \neq 0$. Expressing the normal velocity $v_{n}=-(\partial r / \partial t) \cos \gamma$ in terms of the radial velocity yields the fundamental evolution equation

$$
\frac{\partial r}{\partial t}=-\frac{a^{4} c_{e q} \tilde{\beta} k_{A D}}{k T} \frac{\left(r^{2}+{r^{\prime}}^{2}\right)^{1 / 2}}{r}\left[\frac{r^{2}+2{r^{\prime}}^{2}-r r^{\prime \prime}}{\left(r^{2}+{r^{\prime}}^{2}\right)^{3 / 2}}-\frac{1}{R}\right]
$$

This partial differential equation can be solved numerically given an initial configuration. In order to reproduce the experimental results we assume the dislocation core is located a distance $A=R / 2$ from the center of the circle. We take a special initial condition where the step starts at the core with curvature $\kappa_{c}$, extends to the circle with some arbitrary shape that joins the circle smoothly, and then follows the circle exactly for many turns in the counterclockwise direction as represented in Fig 1 This choice of initial conditions fixes the both the dislocation core and the outer circle throughout the entire evolution, since there are no interactions between different step segments in this model. The boundary condition confining the top step to the outer circle builds in the main effect of step repulsions, which is the physical origin of the confinment.

The numerical solution of the partial differential equation yields a time dependent shape of the spiral that is shown in Fig. 2 $\mathrm{b}$. We find that after a brief transient period, the time-dependent shapes become independent of the initial conditions, and quantitatively reproduce the experimental observations. The marked change in evolution of the step shape for $\theta_{0}$ between $\pi$ and $2 \pi$ is reproduced by these solutions. In this range, the step shape contains highly curved regions as the step passes through the constricted geometry between the dislocation and the facet edge, leading to very large normal velocities in some parts of the step.

The time-period of one rotation for a top facet of radius $385 \mathrm{~nm}$ at a temperature of $390 \mathrm{~K}$ is observed to be 23 minutes. Substituting these values along with the value of the lattice parameter $(a=0.287 \mathrm{~nm})$ into the model gives $c_{e q} k_{A D} \tilde{\beta} / k T=3.9 \times 10^{4} \mathrm{~nm}^{-2} \mathrm{~s}^{-1}$. Using $\tilde{\beta}=0.315 \mathrm{eV} / \mathrm{nm}$ at $390 \mathrm{~K}\left[\underline{6},[26]\right.$ we get $c_{e q} k_{A D}=$
$4.19 \times 10^{3} \mathrm{~nm}^{-1} \mathrm{~s}^{-1}$. This is in good agreement with calculations from previous experiments [18]. The fact that the numerical values are reasonable, along with the excellent agreement with the shape evolution, suggest that the additional mass transport mechanism, step edge diffusion (SED) does not play a major role in the overall evolution. This agrees with our physical expectation that for mass transport over large length scales, SED, as a "one-lane highway", will be ineffective compared to mechanisms (AD or TD) in which material transport occurs across terraces [27].

In order to explore the connection with the growth spirals of Burton, Cabrera and Frank (BCF) [8], we construct approximate solutions called "uniformly rotating shapes" (URS). These shapes have, at any given instant corresponding to a value of $\theta_{0}$, a constant angular velocity $(\omega=d \theta / d t)$ along the step from the core to the point $\mathrm{T}$ where they join the circle smoothly with a continuous first derivative $r^{\prime}$. But unlike the case of a centered spiral, the angular velocity and the total arc length from the core to the joining point will change for solutions at different time instants.

For such URS the left side of Eq. B equals $r^{\prime} \omega$ and the equation reduces to an ordinary differential equation (ODE). Its solution for $r(\theta)$ for a given angle $\theta_{0}$ can be obtained by a shooting method [28] described here. We start with the known values of $r\left(\theta_{0}\right)=0$ and $r^{\prime}\left(\theta_{0}\right)=2 / \kappa_{c}$, pick a particular value of $r^{\prime \prime}(0)$ and numerically integrate the ODE. The value of $r^{\prime \prime}\left(\theta_{0}\right)$ is then adjusted until the solution joins the outer circle with a common tangent. The result for $r(\theta)$ with the smallest absolute value of $r^{\prime \prime}$ gives the URS for the given angle $\theta_{0}$. Since the angular velocity $\omega$ is the same at different points along the step, we can calculate it from a Taylor expansion around $r=0$ with the result $\omega=3 r^{\prime \prime}\left(\theta_{0}\right) a^{4} c_{e q} \tilde{\beta} k_{A D} / 8 R^{3} k T$. Figure 3 shows the URS for the values of $\theta_{0}$ in the time-dependent shapes. The URS joining the circle in the half plane above the diagonal line in Fig. 3 closely resemble the time dependent shapes, and the times predicted using the instantaneous values of $\omega$ are consistent with the time dependent solution. It can be mathematically shown that all the URS have to join the circle in this half plane. Here the distance of step segment from the core increases with increasing arc length along the curve, and a smooth URS satisfying the ODE can be found.

However no such URS exist for a step joining the circle in the half plane below the diagonal line, where there are regions of the step where this radial distance must decrease and $r^{\prime}$ is negative. If we try to define a local angular velocity for the time dependent solutions at each point along the step as the right hand side of Eq. 3 divided by $r^{\prime}$, then this estimate for the angular velocity will diverge in regions when $r^{\prime}$ tends to zero and is negative when $r^{\prime}$ is negative. This shows that the timedependent motion of the step in general cannot be well 


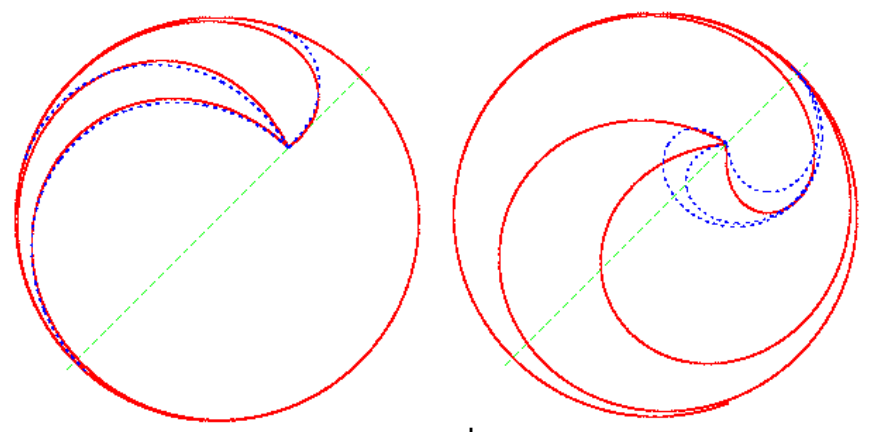

FIG. 3: color on-line.Snapshots of the time dependent evolution (bold red curves) and the URS at the same $\theta_{0}$ (dashed blue curves) superimposed on each other. (a) The shapes calculated at $0 \mathrm{~min}, 8 \mathrm{~min}$, and $22 \mathrm{~min}$ closely match the URS for the same $\theta_{0}$ whereas (b) those at $12 \mathrm{~min}, 18 \mathrm{~min}$, and $21 \mathrm{~min}$ do not resemble the URS at the same $\theta_{0}$. All URS have joining points lying above the diagonal dashed line that passes through the dislocation core and the circle center.

approximated by purely rotational motion.

The ODE is mathematically identical to the equation for spiral growth used by BCF. However, the physical interpretation of the cutoff radius is different from BCF. Unlike the BCF growth spirals, the shape and angular velocity of the URS are determined by the boundary condition at the crystallite edge. Thus the URS represents a different branch of the solution to the ODE. These solutions have a value of curvature at the core that is a local minimum, as opposed a global maximum as is the case for the BCF spirals. Unlike the expanding growth spirals, these solutions, when followed beyond the outer circle boundary, converge to a circle of radius $R$ centered at the core.

In conclusion, STM observations of mass transport via spiral step evolution can be effectively modeled using simple geometric boundary conditions and the standard formulations of the continuum step model. The resulting motion is significantly different from previous theoretical and experimental studies of unconstrained spiral motion, revealing nonuniform angular velocity of the evolving spiral step arising from the asymmetry in the position of the dislocation core with respect to the confining geometry. Mechanisms of mass transport and structural rearrangement in the confined conditions to be expected in nanoscale structures are likely to reveal many more such cases of interesting boundary effects.

Acknowledgments: This work has been supported by NSF-MRSEC at University of MD, DMR \#00-80008. We are grateful to Mr. Chenggang Tao for help with image processing.

* Electronic address: madhav@glue.umd.edu $\dagger$ currently atDepartment of Chemistry, University of Pittsburgh, Pittsburgh, PA 15260.

‡ currently atDepartment of Chemistry, University of Chicago, Chicago, IL 60637.

[1] A. Ichimiya, Y. Tanaka, and K. Ishiyama, Phys. Rev. Lett. 76, 4721 (1996).

[2] D. J. Liu, E. S. Fu, M. D. Johnson, J. D. Weeks, and E. D. Williams, J. Vac. Sci. Technol. B 14, 2799 (1996).

[3] K. Thurmer, J. E. Reutt-Robey, and E. D. Williams, Surf. Sci. 537, 123 (2003).

[4] S. Tanaka, N. C. Bartelt, C. C. Umbach, R. M. Tromp, and J. Blakely, Phys. Rev. Lett. 78, 3342 (1997).

[5] K. Thurmer, J. E. Reutt-Robey, E. Williams, M. Uwaha, A. Emundts, and H. P. Bonzel, Phys. Rev. Lett. 87, 186102 (2001).

[6] D. B. Dougherty, K. Thurmer, M. Degawa, W. G. Cullen, J. E. Reutt-Robey, and E. D. Williams, Surf. Sci. 554, 233 (2004).

[7] F. C. Frank, Disc. Faraday Soc. 5, 48 (1949).

[8] W. K. Burton, N. Cabrera, and F. C. Frank, Philos. Trans. Roy. Soc. London Ser. A 243, 299 (1951).

[9] A. Parkhomovsky, A. M. Dabiran, B. Benjaminsson, and P. I. Cohen, Applied Physics Letters 78, 2315 (2001).

[10] S. Kodambaka, S. V. Khare, W. Swiech, K. Ohmori, I. Petrov, and J. E. Greene, Nature 429, 49 (2004).

[11] N. Cabrera and M. M. Levine, Philos. Mag. 1, 450 (1956).

[12] A. Karma and M. Plapp, Phys. Rev. Lett. 81, 4444 (1998).

[13] T. Surek, J. P. Hirth, and G. M. Pound, J. Cryst. Growth 18, 20 (1973).

[14] G. H. Gilmer and J. D. Weeks, Advances in Chemical Physics, Vol. XL (John Wiley, New York, 1979).

[15] M. Nowicki, A. Emundts, and H. Bonzel, Prog. Surf. Sci. 74, 123 (2003).

[16] M. Nowicki, C. Bombis, A. Emundts, H. P. Bonzel, and P. Wymblatt, New Journal of Physics 4, 60 (2002).

[17] M. Degawa, F. Szalma, and E. D. Williams, Surf. Sci. 583, 126 (2005).

[18] M. Degawa, and E. D. Williams, preprints.

[19] H.Bonzel, Phys. Rep. 385, 1 (2003).

[20] Movies of the step motion can be downloaded from the web at http://www.glue.umd.edu/ jjdw

[21] I. Markov, Crystal Growth for Beginners (World Scientific, Singapore, 1995), 1st ed.

[22] H.-C. Jeong and E. D. Williams, Surf. Sci. Rep. 34, 171 (1999).

[23] The correct physical behavior contains a mixture of rates including slow terrace diffusion. However, as shown in previous work [22, 29], adding that complexity to the modelling only modifies the specific kinetic parameters, but not the qualitative nature of the evolution.

[24] N. C. Bartelt, T. L. Einstein, and E. D. Williams, Surf. Sci. 276, 308 (1992).

[25] N. Israeli and D. Kandel, Phys. Rev. B 60, 5946 (1999).

[26] M. Nowicki, C. Bombis, A. Emundts, and H. P. Bonzel, Phys. Rev. B 67, 075405 (2003).

[27] A. Pimpinelli and J. Villain, Physics of Crystal Growth (Cambridge University Press, United Kingdom, 1998).

[28] W. H. Press, S. A. Teukolsky, W. T. Vetterling, and B. P. Flannery, Numerical Recipes in $C$ (Cambridge University Press, New Delhi, 1992), 2nd ed.

[29] D. J. Liu and J. D. Weeks, Phys. Rev. B 57, 14891 (1998). 